\title{
Dynamics of Asymmetric Nonlinear Vibration Absorber
}

\author{
Chiou-Fong Chung \\ Department of Mechanical Engineering, National Central University, Chung-Li, Taiwan 32054 R.O.C. \\ Chiang-Nan Chang \\ Department of Mechanical Engineering, National Central University, Chung-Li, Taiwan 32054 R.O.C., \\ changcnc@cc.ncu.edu.tw
}

Follow this and additional works at: https://jmstt.ntou.edu.tw/journal

Part of the Mechanical Engineering Commons

\section{Recommended Citation}

Chung, Chiou-Fong and Chang, Chiang-Nan (2003) "Dynamics of Asymmetric Nonlinear Vibration Absorber," Journal of Marine Science and Technology. Vol. 11: Iss. 1, Article 2.

DOI: $10.51400 / 2709-6998.2271$

Available at: https://jmstt.ntou.edu.tw/journal/vol11/iss1/2

This Research Article is brought to you for free and open access by Journal of Marine Science and Technology. It has been accepted for inclusion in Journal of Marine Science and Technology by an authorized editor of Journal of Marine Science and Technology. 


\title{
DYNAMICS OF ASYMMETRIC NONLINEAR VIBRATION ABSORBER
}

\author{
Chiou-Fong Chung and Chiang-Nan Chang*
}

Key words: bifurcation, absorber, harmonic balance, Floquet, subharmonic.

\begin{abstract}
A dynamics of stability and bifurcation analysis of an asymmetrically nonlinear absorber system that contains a main part and a nonlinear spring is proposed. For this study, the analytical works on this nonlinear vibration absorber were performed by the harmonic balance method and the Floquet theory. Dynamic phenomena due to changing of periodic excitation are analyzed. The investigation fulfils that the bifurcation sequences illustrate completely the complex phenomena of system dynamics. Furthermore, this study show that the primary bifurcation orbit coexist with orbit of the secondary responses via a saddle-node bifurcation in a specific period excitation range. The results demonstrate that the new phenomena occur in the strongly nonlinear system.
\end{abstract}

\section{INTRODUCTION}

The computation of optimal design parameters for a type of such linear classical system was discussed by Hartog [3]. However, little work has been done on damped vibration absorbers. Rice and Craith [18] proposed a nonlinear vibration absorber incorporating an asymmetric nonlinear Duffing-type absorber into the system. Nissen et al. [14] found that the bandwidth of a nonlinear vibration absorber with a strongly nonlinear spring is wider than one for an absorber with weakly nonlinear spring. Through Hopf bifurcation, an unstable solution near the operating frequency was generated. The occurrence of this Hopf bifurcation disrupts the performance of the nonlinear vibration absorber due to the high vibration amplitude in this unstable range. In many works, vibration absorbers with a cubic nonlinearity, and with variable rigidity due to the hyperbolic sine law, as well as those with piecewise linear characteristics were investigated $[1,4,16]$. However, the study of the global dynamics behavior of

Paper Submitted 06/03/02, Accepted 07/19/02. Author for Correspondence: Chiang-Nan Chang.

*Department of Mechanical Engineering, National Central University, Chung-Li, Taiwan 32054 R.O.C.E-mail: changcnc@cc.ncu.edu.tw. a nonlinear vibration absorber has been limited.

The jump phenomenon in the Duffing equation is a phenomenon of cubic nonlinearity. A saddle-node bifurcation that a stable periodic orbit and an unstable periodic orbit is coalesced or generated each other are found in the Duffing equation. Some investigations [11, 21] have studied the phenomenon. Meanwhile, the bifurcation is one of routes to chaos [19]. Another saddle-node bifurcation that two unstable period orbits are coalesced or generated each other is found in some nonautonomous systems $[2,5]$, but so far the mechanism of producing such a bifurcation has been poorly understand from a theoretic point of view.

The periodic orbits of period- 1 and the orbits that bifurcate from the periodic orbits mainly construct the dynamics of the system are called as primary responses of the system. The dynamics structure of nonautonomous system that is output of the system may not belong to the primary response $[17,20]$. The output of the system is a subharmonic orbits whose period is a multiple of the period of the periodic excitation. The subharmoic orbits that are called as secondary responses of system. This orbits do not bifurcate from primary response. Furthermore, the subharmonic orbits coexist with the primary response. The subharmonic orbits change their stability due to some bifurcations. Some researches [7, 22] studies the dynamics of the secondary response alone.

The nonlinear behavior of this vibrating absorber is asymmetric nonautonomous system. Periodic orbits of the system are detected by the shooting method [9, 10]. Then the stability of the periodic orbits is performed through Floquet theory $[8,13]$. Based on the parametric continuation algorithm, bifurcation points and bifurcation lines constructed by the bifurcation points are obtained with varying parameters of the nonlinear system. Besides, frequency responses are calculated via the harmonic balance method. The analysis results provide information about the dynamics of the system to study the mechanism of producing the saddle-node bifurcation with the coalescence of two unstable periodic orbits according to the bifurcation 
theory $[6,12]$ and show the coalescence of the primary responses and the secondary responses.

\section{FORMULATION OF THE PROBLEM}

Figure 1 shows a schematic diagram of an asymmetric vibration absorber. The system contains a main rotating part, shown as $m_{1}$, and an absorbing part, shown as $m_{2}$. The force $f(x)$ contains a nonlinear spring, and thus have both linear and nonlinear stiffness. The viscous damper is designed for linearity. The external force comes from an eccentric mass that is fixed on the rotating disk. The equations of motion can be derived as follows:

$$
\begin{aligned}
m_{1} \bar{x}_{1}^{\prime \prime} & +c_{1} \bar{x}_{1}^{\prime}+\left(\bar{k}_{c 1} \bar{x}_{1}+\bar{k}_{c 3} \bar{x}_{1}^{3}\right)-c_{2}\left(\bar{x}_{2}^{\prime}-\bar{x}_{1}^{\prime}\right) \\
- & {\left[\bar{k}_{a 1}\left(\bar{x}_{2}-\bar{x}_{1}\right)+\bar{k}_{a 3}\left(\bar{x}_{2}-\bar{x}_{1}\right)^{3}\right] } \\
& =f_{c o n c}+\bar{m}_{e} R \bar{\omega}^{2} \cos (\bar{\omega} \cdot \tau) \\
m_{2} \bar{x}_{2}^{\prime \prime} & +c_{2}\left(\bar{x}_{2}^{\prime}-\bar{x}_{1}^{\prime}\right)+\left[\bar{k}_{a 1}\left(\bar{x}_{2}-\bar{x}_{1}\right)\right. \\
& \left.+\bar{k}_{a 3}\left(\bar{x}_{2}-\bar{x}_{1}\right)^{3}\right]=f_{\text {conc }},
\end{aligned}
$$

where $\bar{x}_{1}$ and $\bar{x}_{2}$ are the displacements of the main system and the vibration absorber respectively. The parameters of this system are

$m_{1}$ : the mass of the main system,

$c_{1}$ : the damping coefficient of the main system,

$\bar{k}_{c 1}$ : the linear stiffness coefficient of the main system,

$\bar{k}_{c 3}:$ the triple stiffness coefficient of the main system,

$m_{2}$ : the mass of the vibration absorber,

$c_{2}$ : the damping coefficient of the vibration absorber,

$\bar{k}_{a 1}:$ the linear stiffness coefficient of the vibration absorber,

$\bar{k}_{a 3}$ : the triple stiffness coefficient of the vibration absorber,

$\bar{m}_{e}:$ the rotating eccentric mass,

$R:$ the eccentricity,

$\varpi$ : the rotating frequency of mass $\bar{m}_{e}$,

$f_{\text {conc }}$ : the constant force acting on the main system, absorber,

$f_{\text {cona }}$ : the constant force acting on the vibration

where the prime denotes the differential with respect to the time $\tau$. The constant forces acting on the main system and the absorber are the gravity

$$
f_{\text {conc }}=-m_{1} \cdot g
$$

$$
f_{\text {cona }}=-m_{2} \cdot g
$$

The transfer functions of the coordinates $\left(\bar{x}_{1}, \bar{x}_{2}\right)$ and $\left(x_{1}, x_{2}\right)$ are

$$
\begin{aligned}
& \bar{x}_{1}=x_{1}-\delta_{x 1}, \\
& \bar{x}_{2}=x_{2}-\delta_{x 2},
\end{aligned}
$$

The values of $\delta_{x 1}$ and $\delta_{x 2}$ can be determined from the static equilibrium of the system; i.e., $\bar{m}_{e}=0$. The equilibrium equations are

$$
\begin{aligned}
& \bar{k}_{c 1} \cdot \delta_{x 1}+\bar{k}_{c 3} \cdot \delta_{x 1}^{3}=\left(m_{1}+m_{2}+\bar{m}_{e}\right) \cdot g, \\
& \bar{k}_{a 1}\left(\delta_{x 2}-\delta_{x 1}\right)+\bar{k}_{a 3}\left(\delta_{x 2}-\delta_{x 1}\right)^{3}=m_{2} \cdot g,
\end{aligned}
$$

The equations of motion of the system can be rewritten by substituting eqs. (2a), (2b), (3a), (3b) into eqs. (1a) and (1b) as follows:

$$
\begin{aligned}
m_{1} x_{1}^{\prime \prime}+ & c_{1} x_{1}^{\prime}+\left[\bar{k}_{c 1} x_{1}+\bar{k}_{c 3}\left(x_{1}^{3}-3 \delta_{x 1} \cdot x_{1}^{2}+3 \delta_{x 1}^{2} \cdot x_{1}\right)\right] \\
& -c_{2}\left(x_{2}^{\prime}-x_{1}^{\prime}\right)-\left\{\bar{k}_{a 1}\left(x_{2}-x_{1}\right)+\bar{k}_{a 3}\left[\left(x_{2}-x_{1}\right)^{3}\right.\right. \\
& \left.\left.+3\left(\delta_{x 1}-\delta_{x 2}\right)^{2}\left(x_{2}-x_{1}\right)+3\left(\delta_{x 1}-\delta_{x 2}\right)\left(x_{2}-x_{1}\right)^{2}\right]\right\} \\
& =\bar{m}_{e} R \bar{\omega}^{2} \cos \left(\bar{\omega}^{2} \cdot \tau\right), \\
m_{2} x_{2}^{\prime \prime}+ & c_{2}\left(x_{2}^{\prime}-x_{1}^{\prime}\right)+\left\{\bar{k}_{a 1}\left(x_{2}-x_{1}\right)+\bar{k}_{a 3}\left[\left(x_{2}-x_{1}\right)^{3}\right.\right. \\
& \left.\left.-3\left(\delta_{x 2}-\delta_{x 1}\right)\left(x_{2}-x_{1}\right)^{2}+3\left(\delta_{x 2}-\delta_{x 1}\right)^{2}\left(x_{2}-x_{1}\right)\right]\right\} \\
& =0 .
\end{aligned}
$$

We can simplify eqs. (5a) and (5b) by dividing $m_{1}$ and $m_{2}$ respectively

$$
\begin{aligned}
x_{1}^{\prime \prime} & +\widetilde{c}_{1} x_{1}^{\prime}+\left[\widetilde{k}_{c 1} x_{1}+\widetilde{k}_{c 3}\left(x_{1}^{3}-3 \delta_{x 1} \cdot x_{1}^{2}+3 \delta_{x 1}^{2} \cdot x_{1}\right)\right] \\
& -\mu \cdot \widetilde{c}_{2}\left(x_{2}^{\prime}-x_{1}^{\prime}\right)-\mu\left\{\widetilde{k}_{a 1}\left(x_{2}-x_{1}\right)+\widetilde{k}_{a 3}\left[\left(x_{2}-x_{1}\right)^{3}\right.\right. \\
& \left.\left.+3\left(\delta_{x 1}-\delta_{x 2}\right)^{2}\left(x_{2}-x_{1}\right)+3\left(\delta_{x 1}-\delta_{x 2}\right)\left(x_{2}-x_{1}\right)^{2}\right]\right\} \\
& =m_{e} R \varpi^{2} \cos \left(\widetilde{\omega}^{2} \cdot \tau\right), \\
x_{2}^{\prime \prime} & +\widetilde{c}_{2}\left(x_{2}^{\prime}-x_{1}^{\prime}\right)+\left\{\widetilde{k}_{a 1}\left(x_{2}-x_{1}\right)+\widetilde{k}_{a 3}\left[\left(x_{2}-x_{1}\right)^{3}\right.\right. \\
& \left.\left.-3\left(\delta_{x 2}-\delta_{x 1}\right)\left(x_{2}-x_{1}\right)^{2}+3\left(\delta_{x 2}-\delta_{x 1}\right)^{2}\left(x_{2}-x_{1}\right)\right]\right\} \\
& =0,
\end{aligned}
$$

where $\widetilde{c}_{1}=\frac{c_{1}}{m_{1}}, \widetilde{c}_{2}=\frac{c_{2}}{m_{2}}, \widetilde{k}_{c 1}=\frac{\widetilde{k}_{c 1}}{m_{1}}, \widetilde{k}_{c 3}=\frac{\widetilde{k}_{c 3}}{m_{1}}$ 
$\widetilde{k}_{a 1}=\frac{\widetilde{k}_{a 1}}{m_{2}}, \widetilde{k}_{a 3}=\frac{\widetilde{k}_{a 3}}{m_{2}}, m_{e}=\frac{\bar{m}_{e}}{m_{1}}, \mu=\frac{m_{1}}{m_{2}}$

Rescaling the dimension of time, the equations of motion are rewritten as,

$$
\begin{aligned}
\ddot{x}_{1} & +\lambda_{c} \dot{x}_{1}+\left[k_{c 1} x_{1}+k_{c 3}\left(x_{1}^{3}-3 \delta_{x 1} \cdot x_{1}^{2}+3 \delta_{x 1}^{2} \cdot x_{1}\right)\right] \\
& -\mu \cdot \lambda_{a}\left(\dot{x}_{2}-\dot{x}_{1}\right)-\mu\left\{k_{a 1}\left(x_{2}-x_{1}\right)+k_{a 3}\left[\left(x_{2}-x_{1}\right)^{3}\right.\right. \\
& \left.\left.+3\left(\delta_{x 1}-\delta_{x 2}\right)^{2}\left(x_{2}-x_{1}\right)+3\left(\delta_{x 1}-\delta_{x 2}\right)\left(x_{2}-x_{1}\right)^{2}\right]\right\} \\
& =r \omega^{2} \cos (\omega \cdot t) \\
\ddot{x}_{2} & +\lambda_{a}\left(\dot{x}_{2}-\dot{x}_{1}\right)+\left\{k_{a 1}\left(x_{2}-x_{1}\right)+k_{a 3}\left[\left(x_{2}-x_{1}\right)^{3}\right.\right. \\
& \left.\left.-3\left(\delta_{x 2}-\delta_{x 1}\right)\left(x_{2}-x_{1}\right)^{2}+3\left(\delta_{x 2}-\delta_{x 1}\right)^{2}\left(x_{2}-x_{1}\right)\right]\right\} \\
& =0
\end{aligned}
$$

where $\omega_{n}^{2}=\widetilde{k}_{c 1}-2 \delta_{x 1} \widetilde{k}_{c 2}+3 \delta_{x 1}^{2} \widetilde{k}_{c 3}+\mu\left(\widetilde{k}_{a 1}+2\left(\delta_{x 1}-\right.\right.$ $\left.\delta_{x 2}\right) \widetilde{k}_{a 1}+3\left(\delta_{x 1}-\delta_{x 2}\right)^{2} \widetilde{k}_{a 3}, \tau=\omega_{n} \cdot t, \dot{x}_{1}=\frac{x_{1}^{\prime}}{\omega_{n}}, \ddot{x}_{1}=\frac{x_{1}^{\prime \prime}}{\omega_{n}^{2}}$, $\dot{x}_{2}=\frac{x_{2}^{\prime}}{\omega_{n}}, \ddot{x}_{2}=\frac{x_{2}^{\prime \prime}}{\omega_{n}^{2}}, \lambda_{c}=\frac{\widetilde{c}_{1}}{\omega_{n}}, \lambda_{a}=\frac{\widetilde{c}_{2}}{\omega_{n}}, k_{c 1}=\frac{\widetilde{k}_{c 1}}{\omega_{n}^{2}}$,

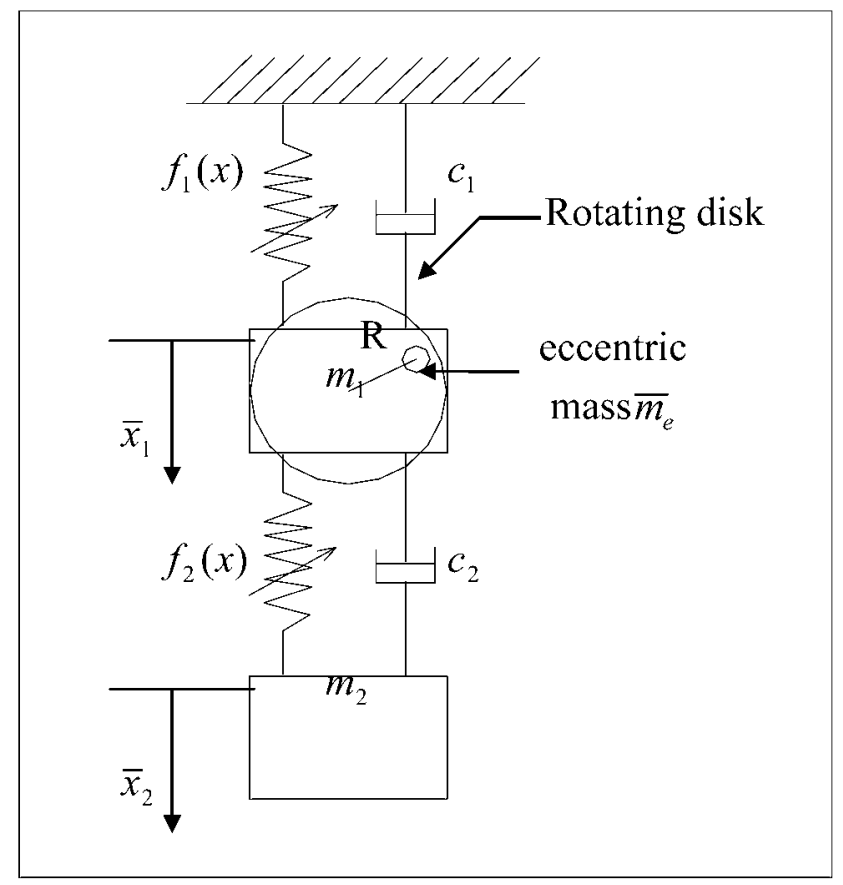

Fig. 1. The schematic diagram of the non-linear dynamic vibration absorber. Where stiffness force $f_{1}(x)=\bar{k}_{c 1} \cdot \bar{x}_{1}+\bar{k}_{c 3} \cdot \bar{x}_{1}^{3}$ and $f_{2}(x)=$ $\bar{k}_{a 1}\left(\bar{x}_{2}-\bar{x}_{1}\right)+\bar{k}_{a 3}\left(\bar{x}_{2}-\bar{x}_{1}\right)^{3}$. $k_{c 3}=\frac{\widetilde{k}_{c 3}}{\omega_{n}^{2}}, k_{a 1}=\frac{\widetilde{k}_{a 1}}{\omega_{n}^{2}}, k_{a 3}=\frac{\widetilde{k}_{a 3}}{\omega_{n}^{2}}, \omega=\frac{\bar{\omega}}{\omega_{n}}, r=m_{e} R$.

\section{THEORETICAL ANALYSIS}

The theoretical analysis includes an approximation of the periodic response, the frequency response and the stability. In order to approximate the periodic response of this system, we employ the analysis method. A nonautonomous system in which a periodic excitation is involved is illustrated as,

$$
\dot{\boldsymbol{x}}=\boldsymbol{F}(\boldsymbol{x}, \omega t),
$$

where $\omega$ denotes the frequency of the periodic excitation. The shooting method $[9,10]$ is an approach of detecting periodic orbits of period-1 and subharmonic orbits of period- $k$ of the system via a Poincaré section that stroboscopically samples a point on a trajectory of the system per period of the orbit. The orbits correspond to fixed points on the section. In the following, the fixed points are detected through a Poincaré map that is mapping of an intersection point of a trajectory with the Poincaré section onto the subsequent intersection point. Selecting some values $\mathrm{y}_{0}$ as the staring point of the procedure of the detection corresponding to the time $t=$ 0 , the Poincaré map $G\left(\mathrm{y}_{0}\right)$ of the point $\mathrm{y}_{0}$ is obtained by numerically integrating eq. (8) with a initial value $\mathrm{y}_{0}$ and computing the solution $\boldsymbol{x}\left(t, \mathrm{y}_{0}\right)$ at the period $k \mathrm{~T}$,

$$
G: \boldsymbol{R}^{\boldsymbol{n}} \rightarrow \boldsymbol{R}^{\boldsymbol{n}} ; y_{0} \rightarrow G\left(y_{0}\right)=\boldsymbol{x}\left(k T, y_{0}\right)
$$

where $T$ is the period of the harmonic excitation. A fixed point $y^{*}$ that corresponds to a periodic orbit or a subharmonic orbit $\overline{\boldsymbol{x}}$ of eq. (8) on the Poincaré section can be determined through zeros of the equation given below,

$$
\hat{G}(y)=G(y)-y .
$$

The orbit $\overline{\boldsymbol{x}}$ is accounted by numerically integrating eq. (8) with the initial value $\mathrm{y}^{*}$ among one period $k \mathrm{~T}$.

The harmonic balance method is another approach to approximate the periodic orbits and subharmonic orbits of the system. The periodic orbit of period-1 or the subharmonic orbit of period- $k$ is approximated by truncated trigonometric functions,

$$
x_{l}(t)=\alpha_{0 l}+\sum_{i=1}^{N} \alpha_{\frac{i}{k} l} \cos \left(\frac{i}{k} \cdot \omega \cdot t+\beta_{\frac{i}{k} l}\right), l=1 \sim n
$$

where $x_{l}$ is the $l$-th element of $\boldsymbol{x}$ and $\alpha_{0 l}, \alpha_{\frac{i}{k} l}$, and $\beta_{\frac{i}{k} l}$ 
are $n \cdot(2 N+1)$ unknown Fourier coefficients. The value of the positive integer $N$ depends on the required accuracy of the approximation. Substitution of eq. (11) into eq. (8) and balancing the coefficients of each of the harmonic terms produce $n \bullet(2 N+1)$ nonlinear algebra equations in terms of the $n \bullet(2 N+1)$ unknown Fourier coefficients. Using those $n \cdot(2 N+1)$ equations, the Fourier coefficients can be numerically calculated. In this study, frequency responses of the system are obtained via the harmonic balance method.

This study obtains the stability of periodic orbits and subharmonic orbits via Floquet theory [8, 13]. Small perturbation to a periodic orbit or a subharmonic orbit determines the stability of the orbit by linearlizing the full equations of motion in relation to the orbit. The result of the linearization is a linear, time variant differential equation.

To perturb a orbit $\overline{\boldsymbol{x}}$, this study substitutes $\boldsymbol{x}=$ $\overline{\boldsymbol{x}}+\widetilde{\boldsymbol{x}}$ into eq. (8). $\widetilde{\boldsymbol{x}}$ represents small perturbation of the orbit $\bar{x}$. The orbit $\bar{x}$ was evaluated through the shooting method or the harmonic balance method which is described in the previous section. Preserving only the linear terms of the equation, the perturbed system can be described as follows,

$$
\dot{\vec{x}}=A(t) \widetilde{\boldsymbol{x}}
$$

where $A(t)$ is a matrix of time-periodic coefficients. The transition matrix $T_{\lambda}(\bar{x})$, which determines the stability of the orbit $\overline{\boldsymbol{x}}$, is calculated numerically from the matrix $A(t)$ with a Runge-Kutta scheme [4]. The characteristic equation of $T_{\lambda}(\overline{\boldsymbol{x}})$ is written as follows,

$$
\begin{aligned}
& \chi(\overline{\boldsymbol{x}}, \lambda, \mu)=\operatorname{det}\left(\mu I-T_{\lambda}(\overline{\boldsymbol{x}})\right) \\
& =\mu^{n}+a_{1} \cdot \mu^{n-1}+\cdots+a_{n-1} \cdot \mu+a_{n}=0,
\end{aligned}
$$

where $I$ is an $n \times n$ identify matrix, $\mu$ is a eigenvalue of the matrix $T_{\lambda}(\overline{\boldsymbol{x}})$, and $\lambda$ is the system parameter. According to the Floquet theory, the orbit $\overline{\boldsymbol{x}}$ is stable if all eigenvalues of its transition matrix have modules less than unity; otherwise it is unstable. A bifurcation occurs in the eigenvalues passing through the unit circle of the complex plane; i.e., one or more the eigenvalues of the transition matrix have unity modulus,

$$
\mu=\cos \theta+j \cdot \sin \theta, 0 \leq \theta \leq 2 \pi,
$$

where $j=\sqrt{-1}$. Three types of instabilities are: (i) $\theta=$ $\pi$, period doubling bifurcation occurs; (ii) $\theta=0$, saddlenode bifurcation, pitchfork bifurcation or transcritical bifurcation occurs; (iii) $\theta \neq 0$ and $\theta \neq \pi$, Hopf bifurcation occurs. Substituting eq. (14) into eq. (13), bifurcation points are obtained by the detection methods and the parametric continuation algorithm [15].

\section{ANALYTICAL RESULTS}

In this study, dynamic phenomena which is due to the changing of periodic excitation is analyzed. Table I shows the fixed parameter values of the eqs. (1a) and (1b). Some investigations [17, 20] found that the subharmonic orbits of the primary responses coexist with the subharmonic orbits of the secondary responses in some nonautonomous systems. Two subharmonic orbits that belong to the same type of the responses are coalesced each other. This study shows a new phenomenon that a subharmonic orbit of the primary responses coalesces with a subharmonic orbit of the secondary responses via a saddle-node bifurcation in a specific parameter range. For analyzing the dynamics of the asymmetric system, this study is performed by using all fixed parameters of eqs. (7a) and (7b) except the forcing frequency $\omega$. Figure 2 portrays a bifurcation diagram on a parameter plane $(\omega, r)$. Figures $3 \mathrm{a}, 3 \mathrm{~b}$, and $3 \mathrm{c}$ portray the frequency response at $r=0.00108$. Bifurcation lines $L_{p d, a}, L_{p d, b}, L_{s n 1, a}, L_{s n 2}, L_{s n 2, a}, L_{s n 2, m a}, L_{s n 2, m b}, L_{H 2, a}$, $L_{H 2, b}, L_{H 2, c}$, and $L_{H 2, d}$ are constructed by period doubling bifurcation points, $P_{p d, a}$ and $P_{p d, b}$, saddle-node bifurcation points, $P_{s n 1, a}, P_{s n 1, b}, P_{s n 2}, P_{s n 2, a}$, and $P_{s n 2, b}$, and Hopf bifurcation points, $P_{H 2, a}, P_{H 2, b}, P_{H 2, c}$, and $P_{H 2, d}$, respectively. Solid lines and dashed lines present the bifurcation lines of subharmonic orbits and those of periodic orbits, respectively. $P_{c 2, a}, P_{c 2, b}, P_{c 2, c}, P_{c 2, d}$ and $P_{c 2, e}$ denote points where a bifurcation line merges with another bifurcation line. To describe transparently the dynamics and to understand clearly the portrayals in the bifurcation diagram, a frequency response along one dash dot line $L_{1}$, are illustrated in the following.

Figure 3(a) illustrates the frequency response of the primary responeses of the coupled asymmetric system at $r=0.00108$. $\alpha_{11}$ and $\alpha_{\frac{1}{2} 1}$ are the Fourier

Table 1. The fixed parameter values of the eqs. (1a) and (1b).

\begin{tabular}{cc}
\hline System parameters & Values \\
\hline$m_{1}(\mathrm{~kg})$ & 1.25 \\
$c_{1}(\mathrm{~N} \bullet \mathrm{s} / \mathrm{m})$ & 0.5 \\
$\frac{k}{k_{c 3}(\mathrm{~N} / \mathrm{m})}$ & 196 \\
$\delta_{x 1}(\mathrm{~m})$ & 98000 \\
$m_{2}(\mathrm{~kg})$ & 0.04608 \\
$c_{2}(\mathrm{~N} \cdot \mathrm{s} / \mathrm{m})$ & 0.6 \\
$\bar{k}_{a 1}\left(\mathrm{~N} / \mathrm{m}^{3}\right)$ & 0.3 \\
${ }_{a 3}\left(\mathrm{~N} / \mathrm{m}^{3}\right)$ & 196 \\
$\delta_{x 2}(\mathrm{~m})$ & 98000 \\
\hline
\end{tabular}


coefficients of a periodic orbit of period-1 and a subharmonic orbit of period-2, respectively. Two saddlenode bifurcation points, $P_{s n 1, a}$ and $P_{s n 1, b}$, divide a resonance of the primary responses into three branches: (i) the upper branch, stable periodic orbits of large amplitude; (ii) the middle branch, unstable periodic orbits; (iii) the lower branch, stable periodic orbits of small amplitude. Meanwhile, a subcritical period doubling bifurcation point $P_{p d, a}$ and a supercritical period doubling bifurcation point $P_{p d, b}$ are on the upper and lower branches, respectively. Subharmonic orbits of period-2 bifurcate from the period doubling bifurcation points $P_{p d, a}$ and $P_{p d, b}$. Furthermore, there are some bifurcations in the subharmonic orbits. The stable subharmonic orbit of period-2 which bifurcates from the period doubling bifurcation point $P_{p d, b}$ and the unstable subharmonic orbit of period-2 which bifurcates from the period doubling bifurcation point $P_{p d, a}$ coalesce each other at a saddle-node bifurcation point $P_{s n 2}$. Besides, the stable subharmonic orbits loss their stability at Hopf bifurcation points $P_{H 2, a}$ and $P_{H 2, b}$. To classify the bifurcation points of the periodic orbits of period- 1 and the points of the subharmonic orbits of period-2, the symbol ' ' denotes the bifurcation points of the periodic orbits of period-1 and the symbol " , represents the bifurcation points of the subharmonic orbits of period-2.

The other responses of the coupled system are the secondary responses as shown in Fig. 3(b). Subharmonic orbits of period-2 are located between saddle-node bifurcation points $P_{s n 2, a}$ and $P_{s n 2, b}$. There are some bifur-

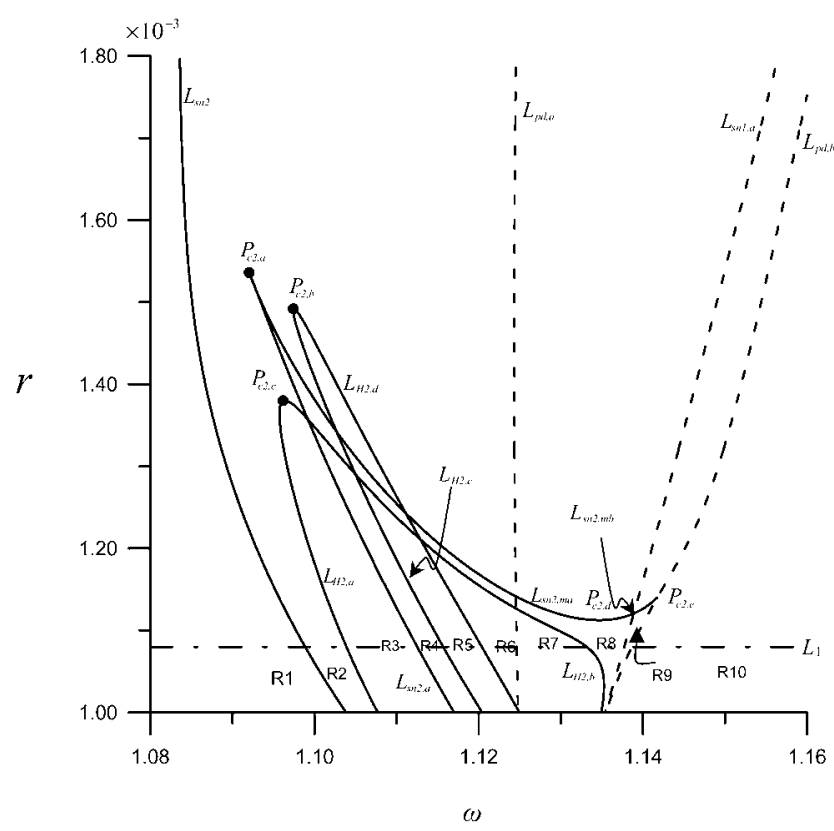

Fig. 2. Bifurcation diagram on a parameter plane $(\omega, r)$. cations of the subharmonic orbits in the figure. The stable subharmonic orbits of the secondary responses change to be unstable at Hopf bifurcation points $P_{H 2, c}$ and $P_{H 2, d}$. The subharmonic orbits coexist with the primary responses as shown in Fig. 3(c) as the descriptions of some investigations $[17,20]$.

\section{SIMULATIONS AND DISCUSSION}

Through the preceding theoretical analysis results, Table 2 and Table 3 show that the bifurcation sequences for $r=0.00108$ by increasing forcing frequency from $\omega$ $=1.09$ of region $\mathrm{R} 1$ to $\omega=1.25$ of region $\mathrm{R} 11$. The bifurcation sequences are shown in Fig. 2. Phase portraits in each region corresponding to that labeled in Fig. 2 are listed in Tables 2 and 3. To verify these theoretical analysis results in $\S 4$, we carry out a series of numerical simulations from eqs. (7a) and (7b). The commercial package DIVPRK of IMSL in FORTRAN subroutines for mathematics applications is used to solve ordinary differential equations. Return to Fig. 2 and Figs. 3a to 3c, we will discuss the bifurcation sequences of regions R1 to R11 again. When the forcing frequency $\omega$ is less than about 1.096, the dynamics of the system shows only a stable period-1. Figure 4 displays the result. As the parameter $\omega$ arrives at $L_{s n 2}$, the stable and the unstable subharmonic orbit of period2 coalesce each other at this saddle-node bifurcation line $L_{s n 2}$. If the forcing frequency $\omega$ passes through regions R1 into R2, a stable periodic orbit of period-1 coexists with a stable subharmonic orbit of period-2, shown as Fig. 5. The converged solution completely depends on initial conditions. Then as the forcing frequency $\omega$ is varied and enters to region R3, a periodic orbit of period-1 still maintain and the stable subharmonic orbit of period-2 undergoes a supercritical Hopf bifurcation line $L_{H 2, a}$ or changes to a torus orbit and an unstable period- 2 orbits. In other words, a period-2 stable subharmonic oscillation loses its stability and becomes a quasi-periodic motion, namely "torus motion" that produces by two incommensurate frequencies, shown as in Fig. 6. On line $L_{S N 2, a}$, the subharmonic orbit of period-2 occur saddle-node bifurcation. As the forcing frequency continues to increase and falls in the region $\mathrm{R} 4$, a stable period orbit of period-1, a quasi-periodic orbit and a stable subharmonic orbit of period-2 are coexistent. In other words, when the forcing frequency $\omega$ varied from region $\mathrm{R} 4$ to region R3, the period-2 stable and unstable subharmonic motions disappear in region R3. These phase portraits of region R4 are shown in Figs. 7a to 7c. In region R5, there exists a period- 1 and two toruses. Beyond supercritical Hopf bifurcation line $L_{H 2, c}$, a period-2 stable subharmonic motion loses its stability and 

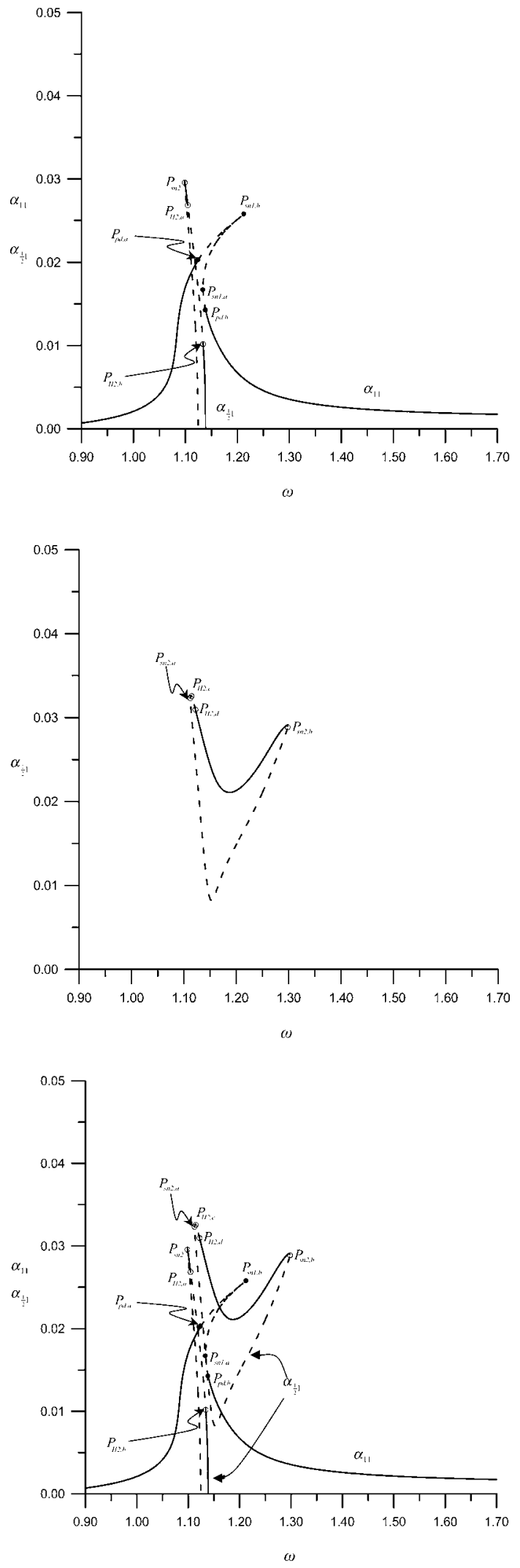

Fig. 3. Frequency response of system: (a) the primary responses, (b) the secondary responses, (c) the coalescence. becomes a quasi-periodic motion, shown in Figs. 8a to 8c. The supercritical Hopf bifurcation appears again at line $L_{H 2, d}$. Due to the supercritical Hopf bifurcation, in region $\mathrm{R} 5$ has one of toruses that comes from a period2 stable subharmonic motion in region R6. Thus, the motions of region R6 have a period orbit of period-1, torus and a period-2 stable subharmonic orbit, shown as in Figs. 9a to 9c. The subcritical period doubling occurs

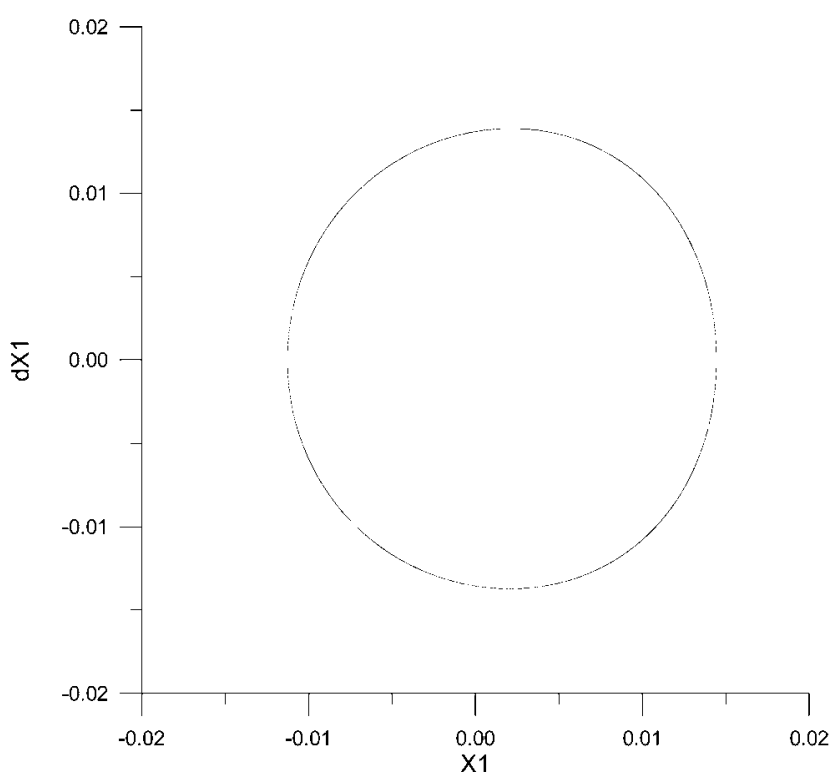

Fig. 4. Phase portrait of region $\mathrm{R} 1$, at $\omega=1.09$, Period- 1 , and $\mathrm{x} 1=x_{1}, \mathrm{dx} 1$ $=\dot{x}_{1}$.

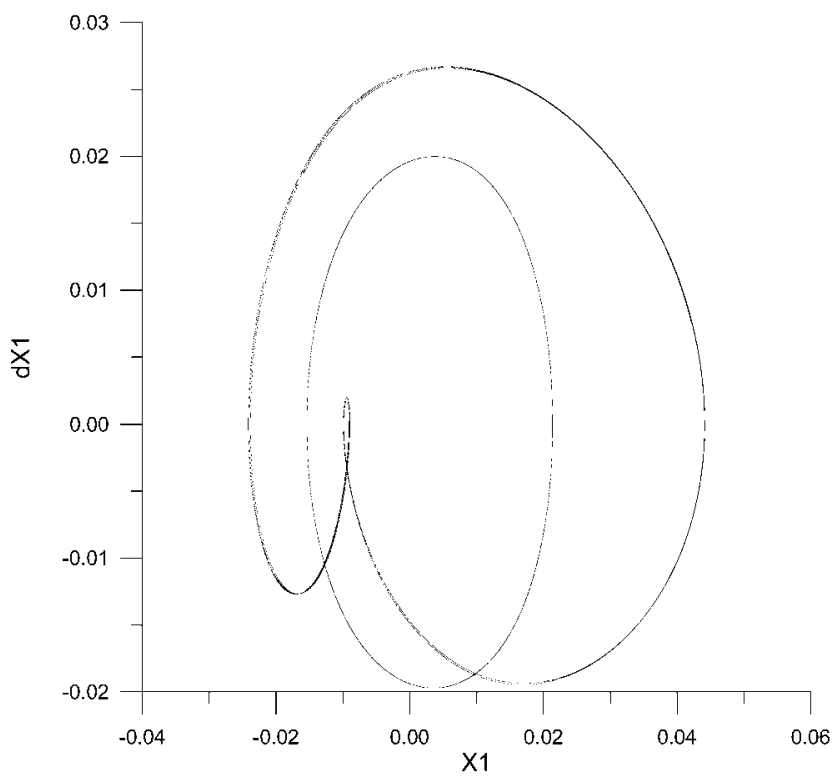

Fig. 5. Phase portrait of region R2, at $\omega=1.1125$, Period- 1 and Period-2, and $\mathrm{x} 1=x_{1}, \mathrm{dx} 1=\dot{x}_{1}$. 
Table 2. Phase portraits in regions $R 1$ to $R 7$ corresponding to that labeled in Fig. 2.

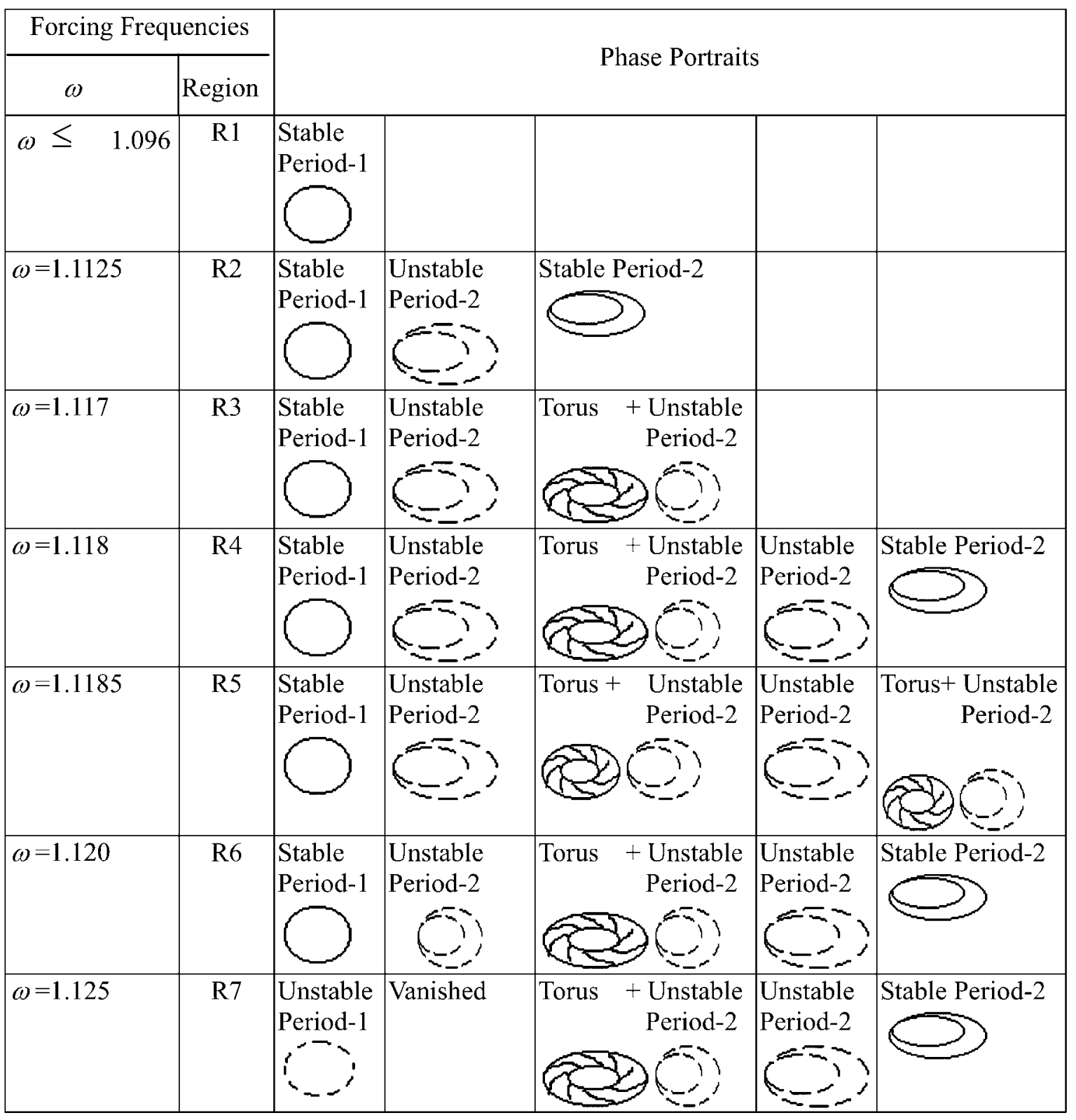

on line Lpd,a. As the forcing frequency varied from region 7 to region 6 , a period-1 unstable harmonic motion of region 7 bifurcates and becomes a stable period- 1 and unstable period- 2 motion of region 6 . In other words, the motions of a stable period-2 and a torus subharmonic orbit are shown in region R7. Figures 10a and $10 \mathrm{~b}$ display the simulation results. On line $L_{H 2, b}$, a supercritical Hopf bifurcation occurs at period-2 subharmonic motion. Hence, as the forcing frequency varied from region $\mathrm{R} 8$ to region $\mathrm{R} 7$, the stable period-2 subharmonic motion changes to be unstable and generates a torus. In region $\mathrm{R} 8$, there exists two distinct stable period-2 subharmonic orbits, shown in Figs. 11a and $11 \mathrm{~b}$. The saddle-node bifurcation line $L_{S N 1, a}$ is constructed by the coalescence of two unstable period orbits. Hence, three unstable period-1 harmonic motions, two stable period-2 subharmonic motions and an unstable period-2 subharmonic motion appear in region R9. The stable orbits shown as in Figs. 12a and 12b. On line $L_{p d, b}$ the supercritical period doubling bifurcation occurs. As the forcing frequency transverse from regions 10 to 9 which crosses line $L_{p d, b}$, a stable period-1 orbit in region 10 losses its stability and becomes to a stable period-2 orbit in region 9 . So, two unstable and 
Table 3. Phase portraits in regions R8 to R11 corresponding to that labeled in Fig. 2.

\begin{tabular}{|c|c|c|c|c|c|c|c|}
\hline \multicolumn{2}{|c|}{ Forcing Frequencies } & \multirow{2}{*}{\multicolumn{6}{|c|}{ Phase Portraits }} \\
\hline \multirow{2}{*}{$\begin{array}{c}\omega \\
\omega=1.135\end{array}$} & \multirow{2}{*}{$\frac{\text { Region }}{\mathrm{R} 8}$} & & & & & & \\
\hline & & $\begin{array}{l}\text { Unstable } \\
\text { Period-1 }\end{array}$ & & & $\begin{array}{l}\text { Stable } \\
\text { Period-2 }\end{array}$ & $\begin{array}{l}\text { Unstable } \\
\text { Period-2 } \\
\quad=-2\end{array}$ & $\begin{array}{l}\text { Stable } \\
\text { Period-2 }\end{array}$ \\
\hline$\omega=1.1385$ & R9 & $\begin{array}{l}\text { Unstable } \\
\text { Period-1 }\end{array}$ & $\begin{array}{l}\text { Unstable } \\
\text { Period-1 }\end{array}$ & $\begin{array}{l}\text { Unstable } \\
\text { Period-1 }\end{array}$ & $\begin{array}{l}\text { Stable } \\
\text { Period-2 } \\
\longrightarrow\end{array}$ & $\begin{array}{l}\text { Unstable } \\
\text { Period-2 } \\
\quad-=\end{array}$ & $\begin{array}{c}\text { Stable } \\
\text { Period-2 }\end{array}$ \\
\hline$\omega=1.20$ & R10 & $\begin{array}{l}\text { Unstable } \\
\text { Period-1 }\end{array}$ & $\begin{array}{l}\text { Unstable } \\
\text { Period-1 }\end{array}$ & $\begin{array}{l}\text { Unstable } \\
\text { Period-1 }\end{array}$ & Vanished & $\begin{array}{l}\text { Unstable } \\
\text { Period-2 } \\
=-2\end{array}$ & $\begin{array}{l}\text { Stable } \\
\text { Period-2 }\end{array}$ \\
\hline $1.22 \leq \omega$ & R11 & Vanished & Vanished & $\begin{array}{l}\text { Stable } \\
\text { Period-1 }\end{array}$ & & $\begin{array}{l}\text { Unstable } \\
\text { Period-2 } \\
\quad \approx-2\end{array}$ & $\begin{array}{l}\text { Stable } \\
\text { Period-2 }\end{array}$ \\
\hline
\end{tabular}

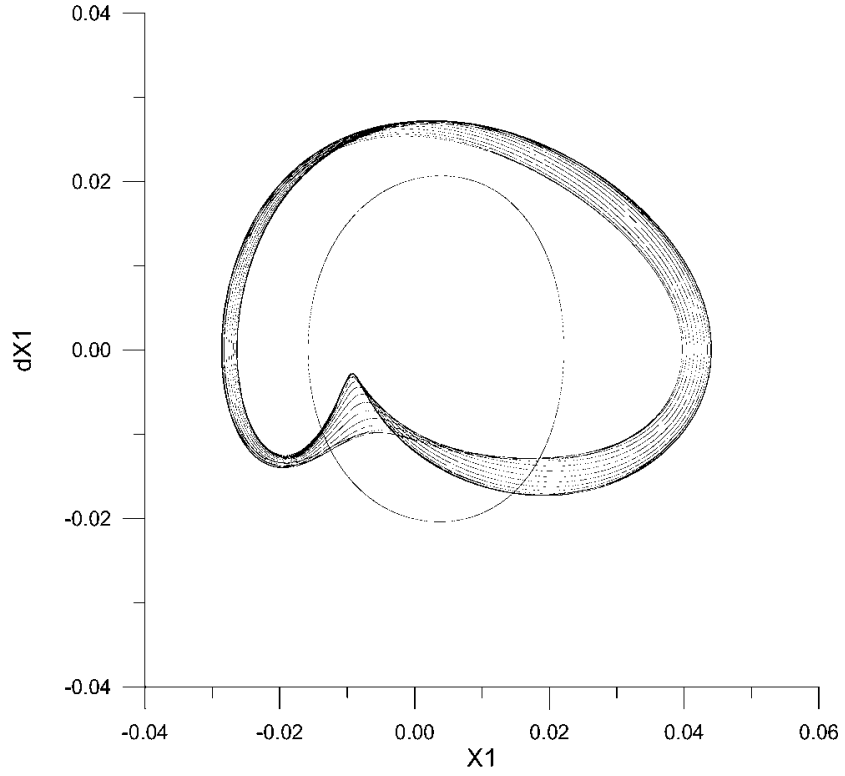

Fig. 6. Phase portrait of region $\mathrm{R} 3$, at $\omega=1.117$, Period- 1 and Torus, and $\mathrm{x} 1=x_{1}, \mathrm{dx} 1=\dot{x}_{1}$. one stable period-1 harmonic motions as well as one unstable and one stable period-2 subharmonic motions exist in region R10 as shown in Fig. 13. Beyond the frequency shown in Fig. 2, region R11 is located in Fig. $3 c$. The forcing frequency is located between points $P_{s n 1, b}$ and $P_{s n 2, b}$. A saddle-node bifurcation is generated by coalescence of two unstable period orbits. As the forcing frequency operated in region R11, Fig. 14 shows a stable period-1 and a stable period-2 subharmonic motion.

\section{CONCLUSIONS}

In this paper, we analyzed a new type of nonlinear vibration absorber with static deflection. It is worth mentioning that when unstable periodic motion occurs in the main rotating system, the dynamics of the system may become complexity and out of control. For the stability analysis, the bifurcation points were obtained and the bifurcation lines were constructed as the parameters were changed. The saddle-node bifurcation line 


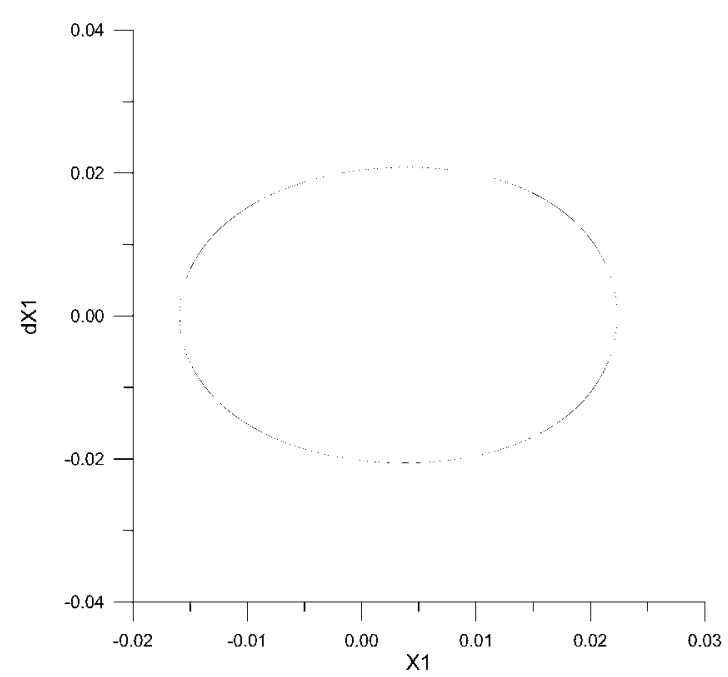

(a)

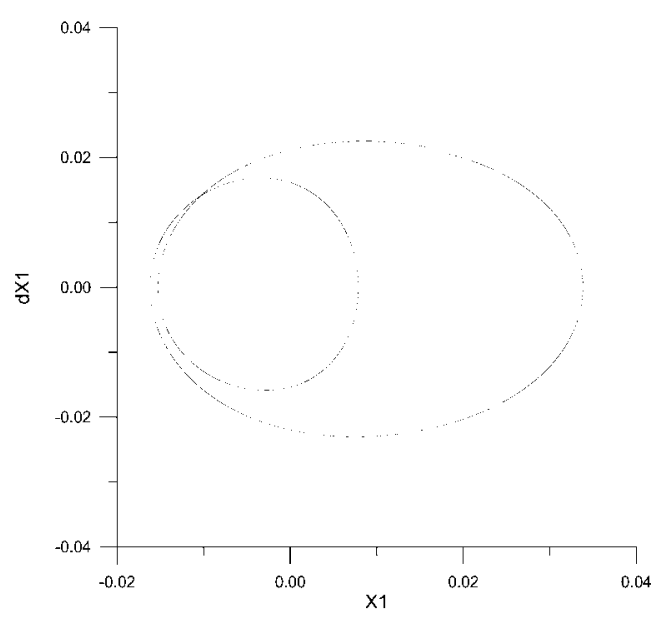

(b)

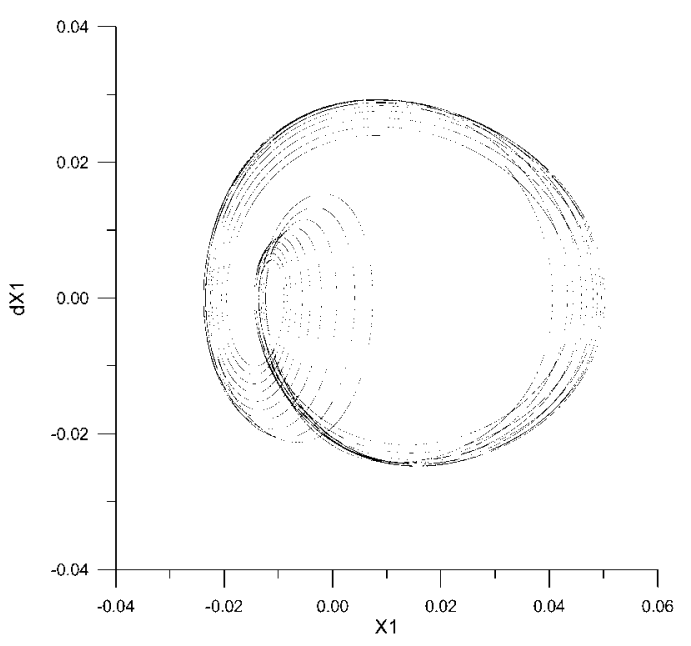

(c)

Fig. 7. Phase portraits of region R4, at $\omega=1.118$ : (a) Period-1, (b) Period2 , (c) Torus, and $\mathrm{x} 1=x_{1}, \mathrm{dx} 1=\dot{x}_{1}$.

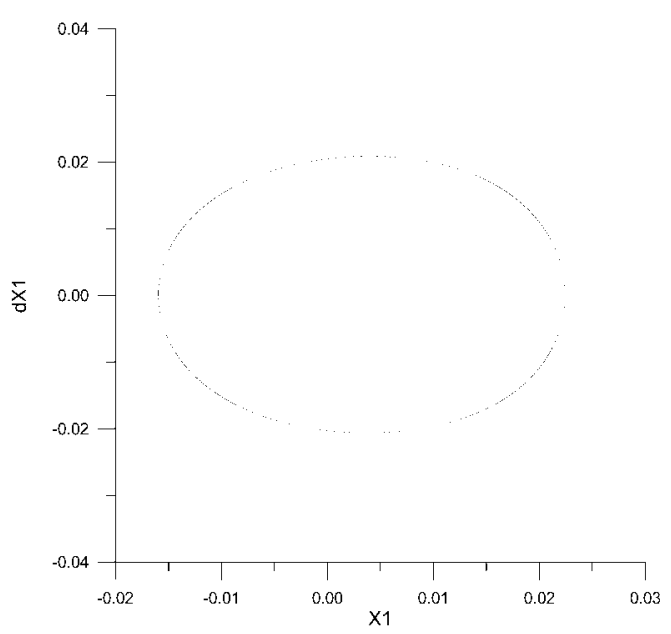

(a)

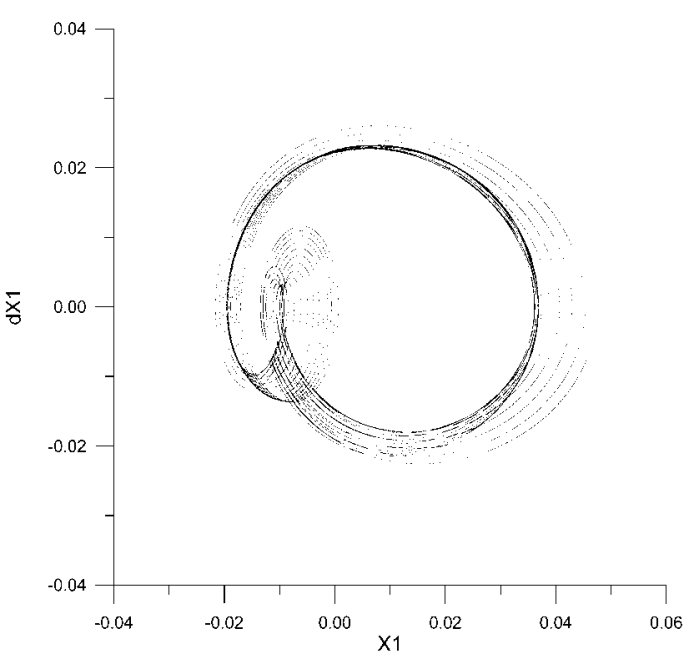

(b)

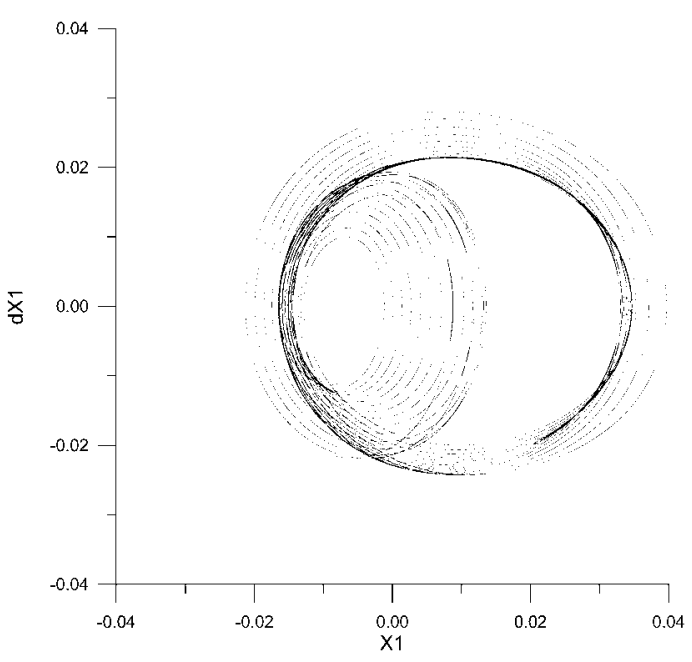

(c)

Fig. 8. Phase portraits of region R5, at $\omega=1.1185$ : (a) Period-1, (b) Torus one, (c) Torus two, and $\mathrm{x} 1=x_{1}, \mathrm{dx} 1=\dot{x}_{1}$. 


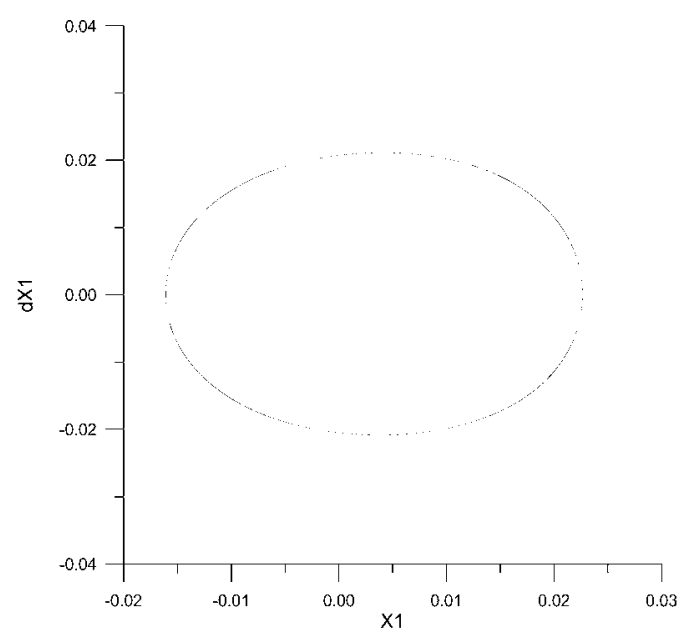

(a)

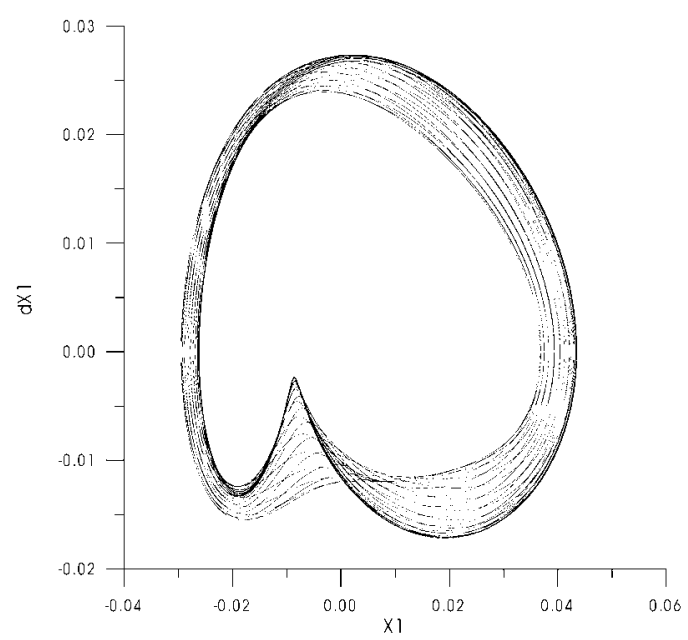

(b)

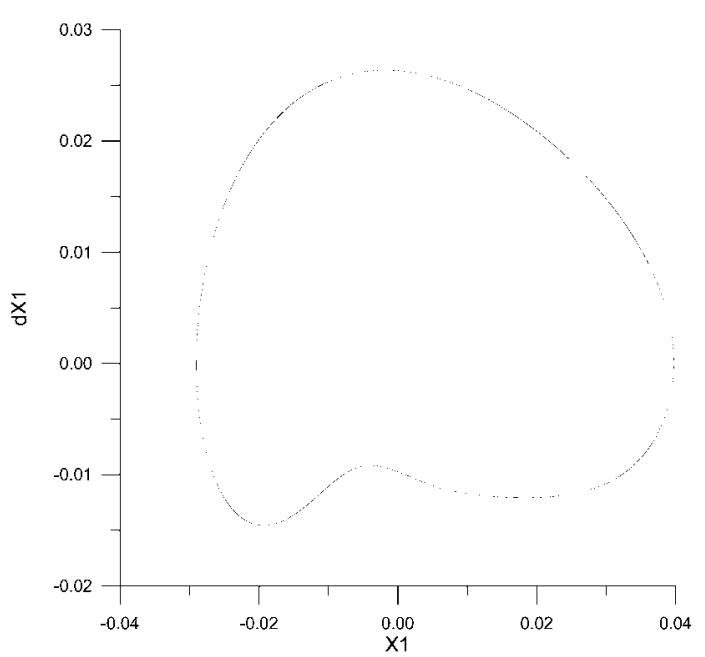

(c)

Fig. 9. Phase portraits of region R6, at $\omega=1.120$ : (a) Period-1, (b) Torus, (c) Period-2, and $\mathrm{x} 1=x_{1}, \mathrm{dx} 1=\dot{x}_{1}$.

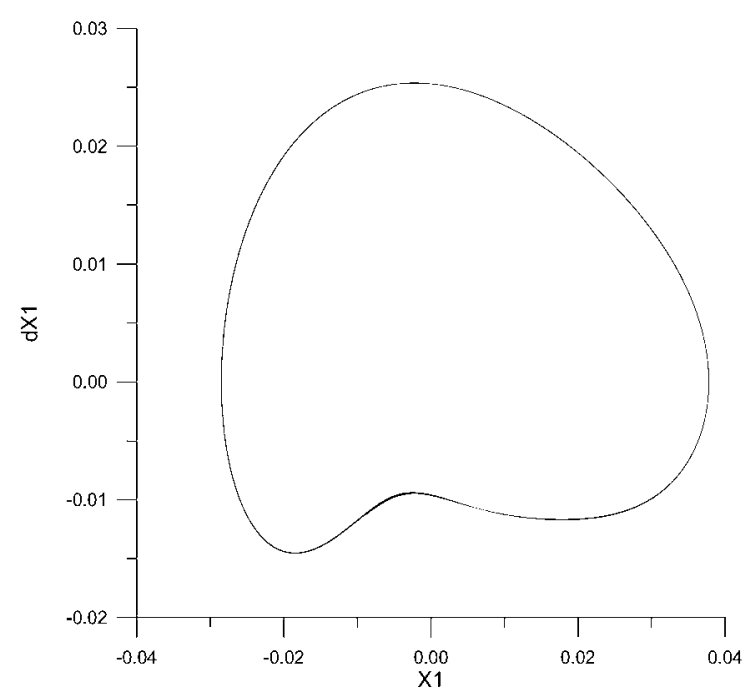

(a)

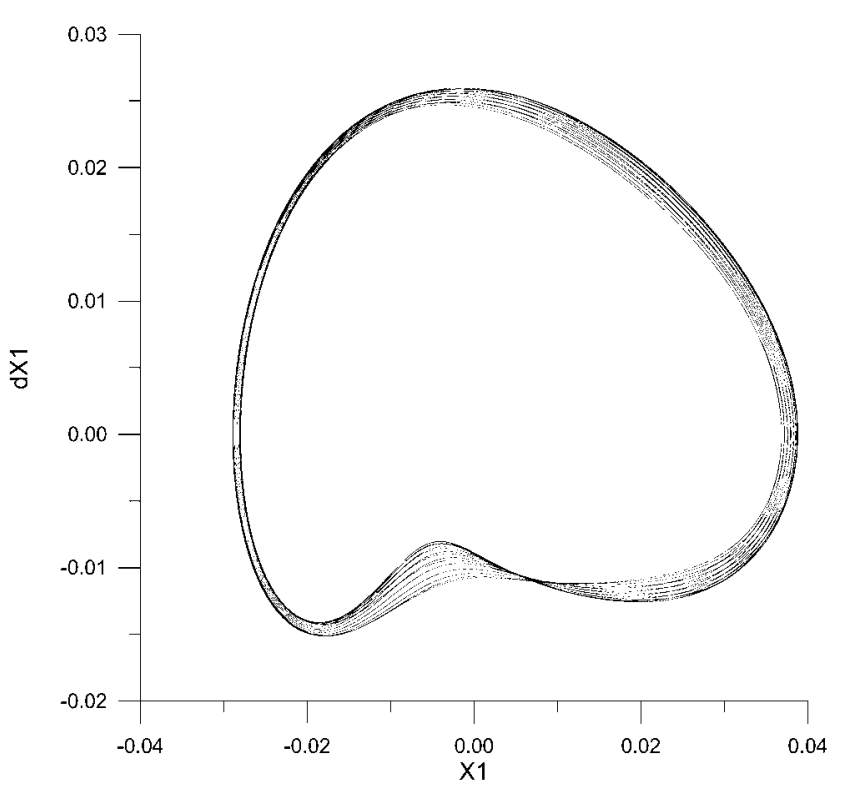

(b)

Fig. 10. Phase portraits of region R7, at $\omega=1.125$ : (a) Period-2, (b) Torus, and $\mathrm{x} 1=x_{1}, \mathrm{dx} 1=\dot{x}_{1}$.

and period doubling bifurcation line are constructed to form a bifurcation set. From the bifurcation set, the mechanism of producing the saddle-node bifurcation with the coalescence of two periodic orbits is clearly illustrated. However, this paper studied that a subharmonic orbit that bifurcates from the primary responses coalesces with a subharmonic orbit of the secondary responses via a saddle-node bifurcation in the nonautonomous system. The investigation fulfils that the bifurcation sequence illustrate completely the complex phenomena of system dynamics. The results dem- 


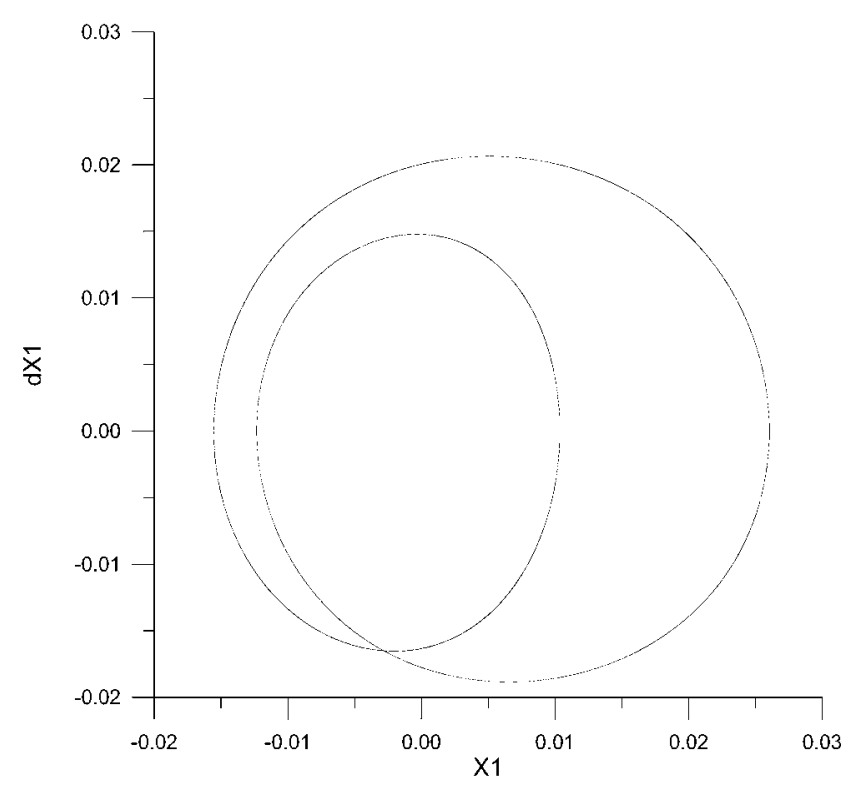

(a)

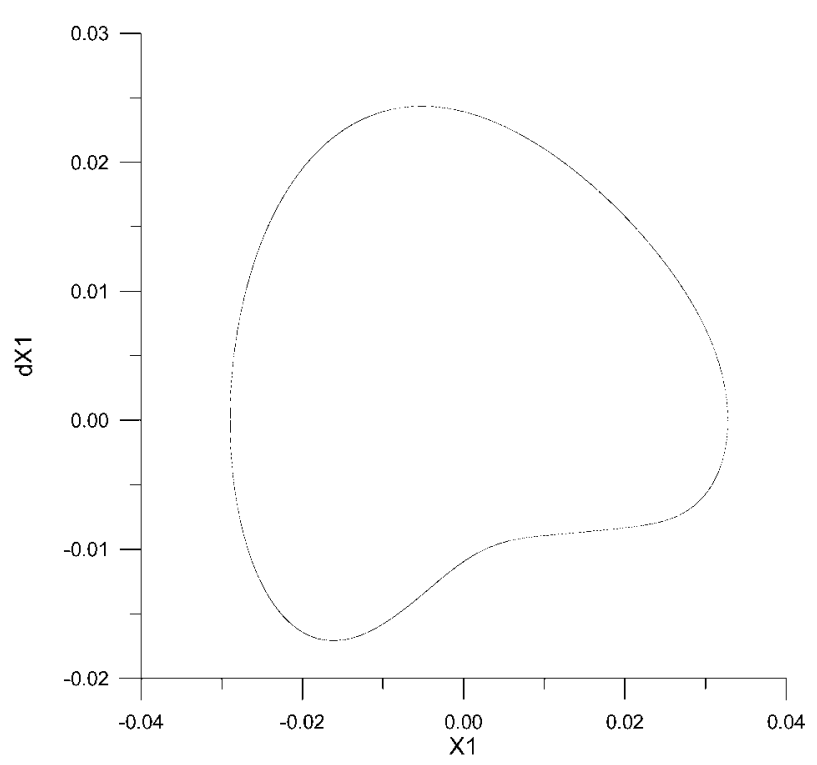

(b)

Fig. 11. Phase portraits of region R8, at $\omega=1.135$ : (a) Period-2, (b) Torus, and $\mathrm{x} 1=x_{1}, \mathrm{dx} 1=\dot{x}_{1}$.

onstrate that the new phenomena occur in the strongly nonlinear system.

\section{REFERENCES}

1. Bauer, H.F., "Steady-State Harmonic and Combination Response of a Nonlinear Dynamic Vibration Absorber," J. Appl. Mech., Vol. 33, pp. 213-216 (1966).

2. Blair, K.B., Krousgrill, C.M., and Farris, T.N., "Non-

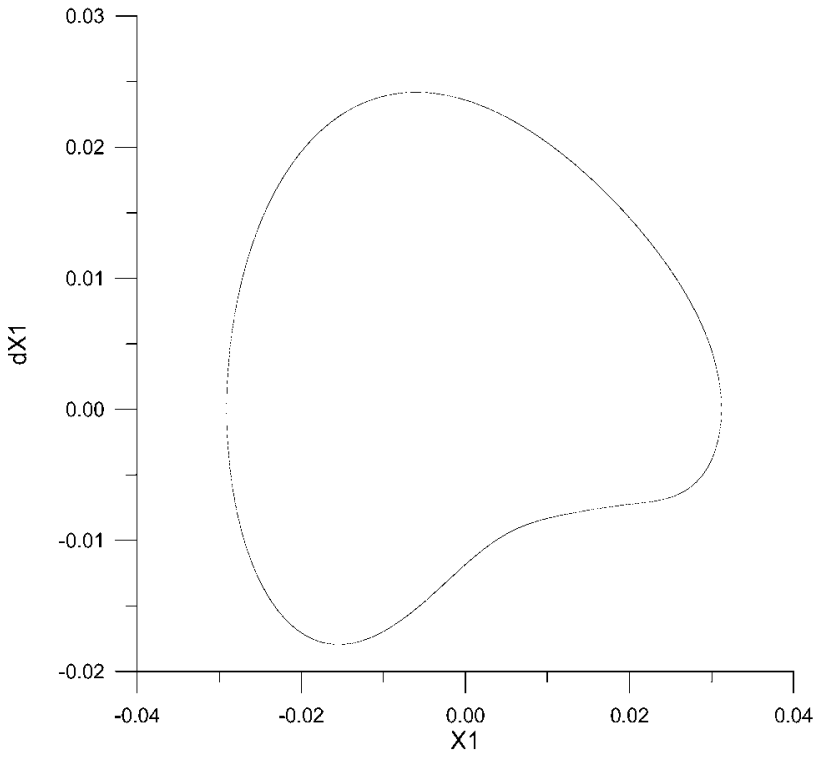

(a)

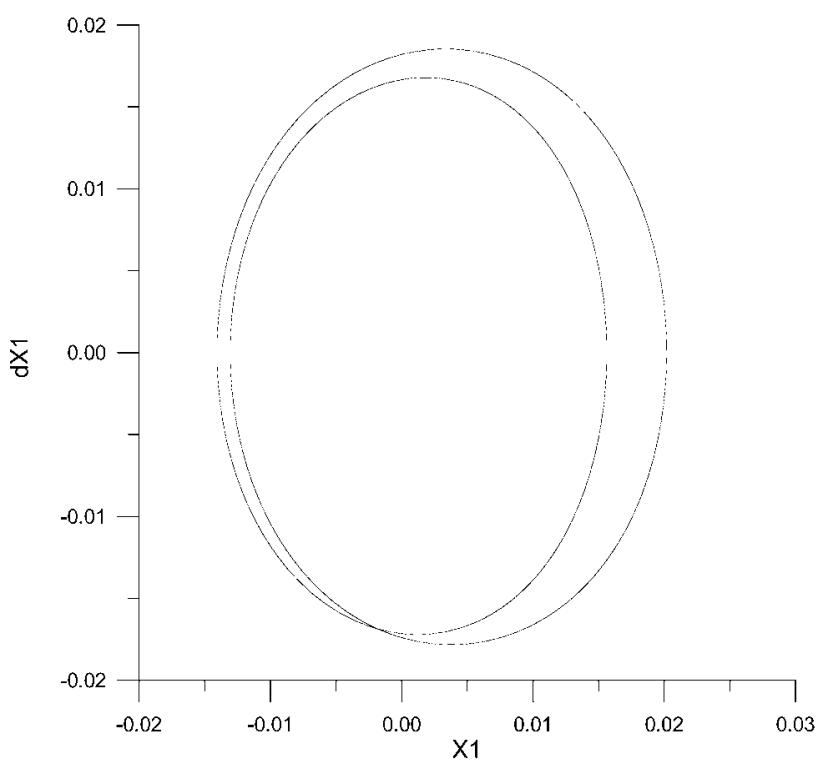

(b)

Fig. 12. Phase portraits of region R9, at $\omega=1.1385$ : (a) Period-2, (b) Period2 , and $\mathrm{x} 1=x_{1}, \mathrm{dx} 1=\dot{x}_{1}$.

Linear Dynamic Response of Shallow Arches to Harmonic Forcing," J. Sound Vib., Vol. 194, No. 3, pp. 353367 (1996).

3. Den Hartog, J.P., Mechanical Vibrations, McGraw-Hill, New York, (1956).

4. Friedmann, P., Hammond, C.E., and Woo, T.H., "Efficient Numerical Treatment of Periodic Systems with Application to Stability Problems," Int. J. Numer. Methods Eng., Vol. 11, pp. 1117-1136 (1977). 


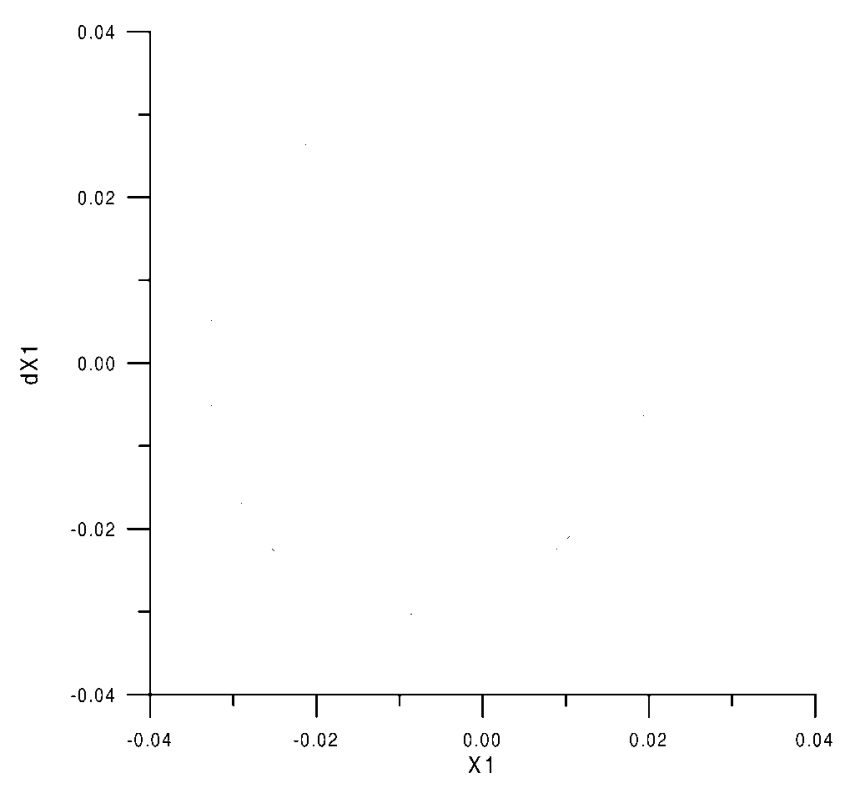

Fig. 13. Phase portrait of region R10, at $\omega=1.20$, Period- 1 and Period-2, and $\mathrm{x} 1=x_{1}, \mathrm{dx} 1=\dot{x}_{1}$.

5. Ge, Z.-M., Yang, H.-S., Chen, H.-H., and Chen, H.-K., "Regular and Chaotic Dynamics of a Rotational Machine with a Centrifugal Governor," Int. J. Eng. Sci., Vol. 37, pp. 921-943 (1999).

6. Guckenheimer, J., and Holmes, P., Nonlinear Oscillations, Dynamical Systems, and Bifurcations of Vector Fields, Springer-Verlag, New York, pp. 376(1983).

7. Holmes, C. and Holmes, P., "Second Order Averaging and Bifurcations to Subharmonics in Duffing's Equation," J. Sound Vib., Vol. 78, No. 2, pp. 161-174 (1981).

8. Hsu, C.S., "Impulsive Parmetric Excitation: Theory," J. Appl. Mech., Vol. 39, pp. 551 (1972).

9. Kawakami, H., "Bifurcation of Periodic Responses in Forced Dynamic Nonlinear Circuits: Computation of Bifurcation Values of the System Paramaters," IEEE Trans. On Circuits and Systems, Vol. CAS-31, No. 3, pp. 248-260 (1984).

10. Kawakami, H. and Yoshinaga T., "Codimension Two Bifurcation and Its Computational Algorithm," Bifurcation and Chaos: Theory and Applications, edited by Awrejcewicz, J., Springer-Verlag, Berlin, New York, pp. 97-132(1995).

11. Kloster, N., and Knudsen, C., "Bifurcations Near 1:2 Subharmonic Resonance in a Structural Dynamic Model," Chaos, Solitons, \& Fractals, Vol. 5, No. 1, pp. 55-66 (1995).

12. Kuznetsov, Y.A., Elements of Applied Bifurcation Theory, Springer-Verlag, New York, (1995).

13. Nayfeh, A.H., and Mook, D.T., Nonlinear Oscillations, John Wiley \& Sons, New York, (1979).

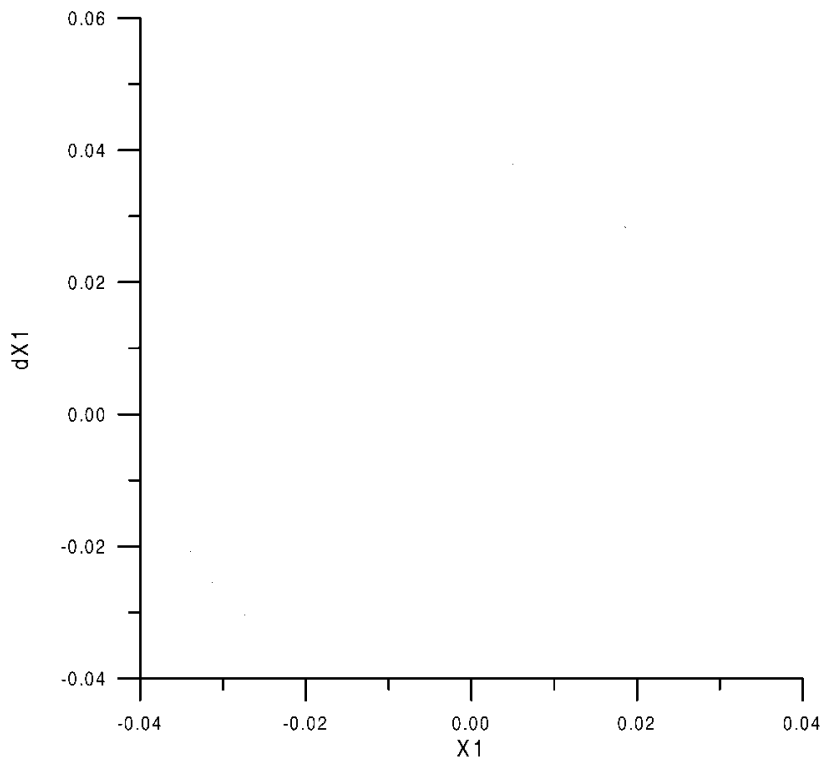

Fig. 14. Phase portrait of region R11, at $\omega=1.25$, Period- 1 and Period-2, and $\mathrm{x} 1=x_{1}, \mathrm{dx} 1=\dot{x}_{1}$.

14. Nissen, J.C., Popp, K., and Schmalhorst, B., "Optimization of a Non-Linear Dynamic Vibration Absorber," $J$. Sound Vib., Vol. 99, No. 1, pp. 149-154 (1985).

15. Padmanabhan, C. and Singh, C., "Analysis of Periodically Excited Non-Linear Systems by a Parametric Continuation Technique," J. Sound Vib., Vol. 184, No. 1, pp. 35-58 (1995).

16. Potekhin, A.F. and Frenke, Ye.I.: Tr. Tambovskogo in Takhim. Mashino-Stroyeniya (1969) 149.

17. Raghothama, A. and Narayanan, S., "Bifurcation and Chaos of an Articulated Loading Platform with Piecewise Non-Linear Stiffness Using the Incremental Harmonic Balance Method," Ocean Eng., Vol. 27, pp. 1087-1107 (2000).

18. Rice, H.J. and Mc Craith, J.R., "Practical Non-Linear Vibration Absorber Design," J. Sound Vib., Vol. 116, No. 3, pp. 545-559 (1987).

19. Schuster, H.G., Deterministic Chaos: An Introduction, Physik-Verlag, Weinheim, p. 126 (1984).

20. Shaw, S.W., "The Dynamics of a Harmonically Excited System Having Rigid Amplitude Constraints," J. Appl. Mech., Vol. 52, pp. 453-458 (1985).

21. Szemplinska-Stupnicka, W. and Rudowski, J., " Local Methods in Predicting Occurrence of Chaos in TwoWell Potential Systems: Superharmonic Frequency Region," J. Sound Vib., Vol. 152, No. 1, pp. 57-72 (1992).

22. Yagasaki, K., "Higher-Order Averaging and UltraSubharmonics in Forced Oscillators," J. Sound Vib., Vol. 210, No. 4, pp. 529-553 (1998). 